\title{
Aprendizaje basado en el modelo STEM y la clave de la metacognición
}

\author{
Learning based on the STEM model and the key of meta-cognition
}

\author{
(D) José Manuel Bautista-Vallejo \\ Departamento de Pedagogía, Universidad de Huelva, España
bautista@uhu.es
}

RECIBIDO 3/8/2019 ACEPTADO 19/1/2020 PUBLICADO 1/6/2020

\section{(D) Rafael Manuel Hernández-Carrera}

Departamento de Didáctica y Organización Escolar, Universidad Internacional de la Rioja, España

rafael.hernandez@unir.net

\section{RESUMEN}

En este trabajo se aborda cómo, a lo largo de los últimos años, la investigación educativa ha contribuido a cambiar la perspectiva de la enseñanza-aprendizaje. Para ello se estudia el proceso de adquisición de conocimientos, destrezas y actitudes, realizando una aproximación epistemológica al modo en que las personas aprenden, y cómo deben adaptarse en función de ello los métodos, los procedimientos y las técnicas didácticas. Se realiza una conceptualización de la inteligencia a tenor de las investigaciones realizadas en los últimos años, así como de nuevas formas de enseñanza-aprendizaje basadas en el modelo STEM (science, technology, engineering and mathematics) como forma de integrar estas áreas de aprendizaje de un modo eminentemente práctico, centrado en la experiencia de docentes y discentes, desplazando la mera adquisición de conocimientos. Todo ello se enmarca en un proceso de aprendizaje metacognitivo cuya finalidad es que el alumno aprenda a aprender, integrando aspectos como el aprendizaje significativo, la metamemoria, la metacomprensión, la metalectura y la metaescritura. El tipo de aprendizaje y competencias necesarias para el futuro es desconocido, por lo que se hace necesaria una reflexividad continua sobre los contenidos y las metodologías de aprendizaje, aspecto que pasa por un cambio curricular y metodológico que tenga presente distintos contextos y modelos de aprendizaje.

PALABRAS CLAVE educación stem, metacognición, proceso de enseñanza-aprendizaje, estrategias educativas.

\section{ABSTRACT}

This work deals with how, over the last few years, educational research has contributed to changing the perspective on teaching-learning process. To this aim, the process of acquiring knowledge, skills, and attitudes are studied, making an epistemological approach to how people learn and how methods, procedures and didactic techniques should be adapted accordingly. Intelligence is conceptualized on the basis of the research carried out in recent years, as well as new forms of teaching-learning based on the STEM model (science, technology, engineering and mathematics) as a way of integrating these learning areas in an eminently practical way, centered on the experience of teachers and learners, displacing the mere acquisition of knowledge. All this is framed within a meta-cognitive learning process whose purpose is that the student learns to learn, integrating aspects such as significant learning, meta-memory, meta-comprehension, meta-reading and meta-writing. The type of learning and skills needed for the future is unknown, which makes it necessary to continuously reflect on the contents and methodologies of learning, an aspect that involves a curricular and methodological change that takes into account different learning contexts and models.

KEYWORDS digital competence/skill, gender, secondary education, girls, stem, didactic methods, gender stereotypes. 


\section{INTRODUCCIÓN}

A lo largo de los últimos años se ha producido un gran cambio en el modo en que el profesorado desarrolla su labor docente. Una forma de plantear esta labor es entender que en primer lugar se debe tener claro cómo aprende el alumnado y, en función de ello, adaptar los métodos, procedimientos y técnicas didácticas.

Una de las consecuencias de esto es el replanteamiento del concepto de inteligencia en la escuela a tenor de las investigaciones desarrolladas a lo largo de los últimos decenios (Ott y Michailova, 2018), así como de las aportaciones realizadas desde la neurociencia y la metacognición (Eysenck, 2018).

En este trabajo abordamos cómo, a lo largo de los últimos años, la investigación educativa ha contribuido a cambiar la perspectiva sobre lo que el profesor debe enseñar a sus alumnos, así como el proceso de adquisición de competencias por parte de estos. En otras palabras, se aborda la cuestión de cómo se aprende, algo que condiciona notablemente los procesos de enseñanza-aprendizaje que tienen lugar en el aula. En definitiva, se abordan los elementos del proceso metacognitivo dado que este es fundamental para el alumnado, puesto que se asocia al aprendizaje autónomo en contextos escolares y desarrollo de competencias profesionales.

Adicionalmente, el objetivo de este artículo es plasmar el interés que la institución educativa manifiesta sobre nuevas formas de enseñanza y aprendizaje en el marco del modelo denominado STEM (siglas en inglés para science, technology, engineering, mathematics), es decir, el modelo dedicado al aprendizaje integrado de las ciencias, la tecnología, la ingeniería y las matemáticas. Se trata de "nuevas" formas de enseñanza basadas en una metodología eminentemente práctica, donde la experiencia del discente desplaza de esta manera a la mera asimilación de conocimientos en compartimentos estancos, con un modelo educativo centrado en la capacidad de innovar, inventar y resolver los problemas de forma creativa, algo que, previsiblemente, las profesiones del futuro van a exigir (Murcia y Pepper, 2018; Sivaraj et al., 2019; Snow y Kaplan, 2018).

En este marco se insiste que es necesario que los discentes aprendan bajo procesos de metacognición o, dicho de otro modo, que el alumnado aprenda a aprender (Gargallo López et al., 2020; Villota Hurtado, 2018). Este proceso influye tanto en la manera de enseñar del profesorado como en la forma de aprender por parte del alumnado.

La investigación sobre metacognición cumple algo más de cuatro décadas. A pesar de ello, aún existen discrepancias sobre su definición y sobre los mecanismos de procesamiento involucrados en ella.

Flavell, al proponer el concepto metacognición, enfatiza en las propiedades del prefijo meta (sobre, o más allá, o más arriba), aludiendo a la capacidad de pensar sobre el pensamiento (Flavell, 1976, 1979) o de una cognición sobre la cognición (Flavell, 1992; Wellman, 1985), la cual consiste en el monitoreo, regulación y orquestación de los procesos cognitivos al servicio de una meta u objetivo (Flavell, 1976).

La metacognición ha tomado una presencia cada vez más importante en el mundo educativo ya que supone analizar cómo aprende el alumno (Perry et al., 2018). Consiste en conocer las estrategias de aprendizaje que utiliza el ser humano y, en base a ellas, poder emplear un diseño didáctico en consonancia. También supone conocer qué estrategias son eficaces y útiles y qué otras no lo son tanto. En este sentido, la promoción de aquellas metodologías que llevan a aprender haciendo son introducidas de forma prioritaria (Godhe et al., 2019).

Por otra parte, la metacognición estratégica sería la capacidad que tenemos como seres humanos de autorregular o autocontrolar nuestro propio aprendizaje; en otras palabras, saber qué tipo de estrategias 
debemos emplear en cada momento, aplicarlas, evaluar su eficacia, controlar el proceso... (Cerezo et al., 2019). Por tanto, debemos reflexionar como docentes sobre qué es lo que hace el alumnado cuando su aprendizaje es eficaz y qué hace o no cuando no lo es.

\section{DESCRIPCIÓN DE LA METACOGNICIÓN}

Profundizar la relación metacognición-STEM hace imprescindible un recorrido a través de la metamemoria, la metacomprensión, la metaatención, la metalectura y la metaescritura.

\subsection{Metamemoria}

Para Dzib-Goodin et al. (2017), desde el punto de vista evolutivo, el proceso de la memoria se desarrolló a nivel cognitivo, sobre todo en los mamíferos, como necesidad para concebir el futuro, empleando elementos conocidos con el fin de responder al ambiente. De ahí su relación tan estrecha con otros procesos como el aprendizaje, el sueño, el movimiento y la atención, pues los recuerdos se crean con fines de pervivencia de las especies. Los elementos conocidos tenían la probabilidad de ocurrir nuevamente, lo cual implica la materia prima para la capacidad de construir nuevos escenarios para la resolución de problemas en el presente (Riley y Constantinidis, 2016).

Dzib-Goodin et al. (2017) explican que en ese desarrollo evolutivo dicho proceso era importante para casi todas las especies, sobre todo cuando la capacidad de movimiento se sofisticó, ya que tanto las primeras especies como los primeros seres humanos requerían de realizar viajes más o menos cortos, contando con puntos de referencia, para aprovechar los recursos (alimentos, agua y refugio), al mismo tiempo que debían evitar a los depredadores.

Este no es un simple detalle en el proceso evolutivo. Estos movimientos o viajes requirieron de un proceso que permitiera las asociaciones de recursos-lugar-señal de peligro y las relaciones espaciales entre estos puntos de referencia de dichas exploraciones. Más tarde, todas estas asociaciones y relaciones espaciales, probablemente también temporales, se volvieron emocionalmente significativas.

En consecuencia, estos viajes pueden haber impulsado la evolución tanto de la memoria espacial como episódica. Sin embargo, esto requiere el reconocimiento de un entorno dinámico (Allman y Mareschal, 2016; Llewelyn y Hobson, 2015), ya que los recursos-lugar y demás asociaciones son susceptibles de cambio y demandan constantemente nuevos puntos de referencia, ricos en señales naturales que pueden ser descubiertos sin mucho trabajo cognitivo, lo que implica una necesidad de crear un recableado de redes neuronales flexible para sobrevivir ante tales circunstancias.

La explicación de estos procesos a partir de los años 70 lleva a la necesidad de estudiar los fenómenos subyacentes a la cognición. Tulving y Madigan (1970), a principios de esa década, refiriéndose al funcionamiento de los propios procesos de memoria, proponen el concepto de metamemoria, antecedente próximo de la metacognición.

La metamemoria, en este sentido, se refiere a los procesos y estructura por el que las personas son capaces de examinar el contenido de sus memorias, a futuro o de manera retroactiva, haciendo juicios o comentarios acerca de ellos (Metcalfe y Dunlosky, 2008). Es decir, con el término se alude al grado de conocimiento y conciencia que posee el individuo acerca de la memoria y de todo aquello relevante para el registro, almacenamiento y recuperación de la información (López Mejías et al., 2017). 
Resulta de interés, entonces, toda indagación en los estudiantes para comprobar si realmente las diferentes modalidades de enseñanza y estilos de aprendizaje propician en ellos la reflexión acerca de sus procesos mnémicos, de las estrategias y la efectividad de los mismos en el aprendizaje (Aguilera Morales et al., 2018). Ante la inclusión del modelo STEM, basado en el aprendizaje integrado de las disciplinas científicas a las que responde el mencionado acrónimo, en donde los procesos de enseñanza y aprendizaje descansan en metodologías activas, manipulativas, constructivistas y por descubrimiento, esta cuestión cobra una especial importancia (Arabit García y Prendes Espinosa, 2020).

\subsection{Aprendizaje significativo}

Agra et al. (2019) proponen un actualizado análisis del concepto del aprendizaje significativo a la luz de la teoría de Ausubel y los usos de otros autores para explicar el conjunto de acciones que el alumnado lleva a cabo en relación a la misma.

La teoría del aprendizaje significativo fue desarrollada por David Ausubel en 1963, lo cual nos da una idea de la gran consistencia que tiene la misma y justifica su fuerza explicativa (Rodríguez Palmero, 2004). Supone no aprender todo memorísticamente sino según nuestro esquema conceptual y cognitivo propio, de manera que las cosas que aprendemos y memorizamos tengan significado para nosotros mismos.

El marco de la integración del modelo STEM y su relación con la metacognición es de especial interés en este ámbito. El aprendizaje significativo, tanto en su vertiente social, como socio-humanística o comportamental, es un marco idóneo para estas metodologías activas, manipulativas, constructivistas y por descubrimiento. Las revisiones más actuales de esta característica del aprendizaje nos llevan a considerarlo como (Agra et al, 2019):

a. La expansión de la estructura cognitiva a través de la incorporación de nuevas ideas que se relacionan con las ideas preexistentes.

b. la existencia de conocimiento estructurado que [...] permite la conexión con el nuevo conocimiento [...] y conecta conocimiento con el que tiene la intención de absorber.

c. La nueva información adquiere significado en la estructura cognitiva [...] interacción entre un nuevo conocimiento y el anterior.

d. La interacción de un nuevo material con lo que ya existe en la estructura cognitiva.

e. El modelo [...] que expande el conocimiento a través de la asimilación de nuevos conceptos con los preexistentes [...] que sirven de base para incorporar, comprender y fijar nuevos conocimientos en el conocimiento cognitivo que estructura el aprendiz.

f. La nueva información [...] genera sentido y significado [...] en aspectos relevantes de la estructura cognitiva.

Por tanto, en el aprendizaje significativo el alumnado pone en relación la información nueva con el conocimiento previo, produciéndose una reestructuración, un reajuste y una reconstrucción del conocimiento. En este sentido, el proceso metacognitivo es fundamental para el alumnado, puesto que se asocia al aprendizaje autónomo en contextos escolares y desarrollo de competencias profesionales. No son pocos los autores que se han hecho eco de esta relación fundamental (Soodla et al., 2017; Spruce y Bol, 2015; Tanner, 2012; Zepeda et al., 2015). 


\subsection{Metacomprensión}

La comprensión es el fin fundamental del aprendizaje (De Bruin et al., 2011). La metacomprensión, por su parte, es el conocimiento de la propia comprensión y de los procesos mentales necesarios para conseguirla y está muy relacionada con los conocimientos previos que tengamos sobre un tema.

Para Gray et al. (2018), en el marco de una reflexión sobre la reforma del currículum en las escuelas europeas y el diseño de entornos de aprendizaje adecuados para generar desde ese nuevo currículum, la metacomprensión se refiere a la capacidad del alumnado para monitorear el grado en que comprenden la información que se les está comunicando, reconocer los fallos en la comprensión y emplear estrategias de reparación.

En este sentido, la metacomprensión supone ser conscientes de hasta qué nivel se comprende lo que podría ser el proceso hasta el logro. Así, el papel de los educadores en la asimilación de esta comprensión es fundamental. Aspectos tales como preguntas que se formulan en el marco de la relación educativa docentediscente pueden determinar un tipo de comprensión. O, por otro lado, la exigencia docente de una reproducción literal de una determinada información (texto), o pedir la elaboración del mismo y la extracción de conclusiones, etc., todo ello puede disponer diferentes niveles y tipos de comprensión en su alumnado.

\subsection{Metaatención}

La atención es un proceso conductual y cognitivo, un proceso psicológico complejo y socialmente mal comprendido, como afirma Dzib Goodin (2013a; 2013b). En este proceso nuestros sentidos se enfocan en una sola cosa, selectivamente. Es algo determinante para la conservación de las especies y que depende de la adaptación al medio. Como proceso está producido por la interconexión de las redes neuronales que permiten un procesamiento en paralelo, secuencial o en base a jerarquías que resulta de la actividad mental con una riqueza y flexibilidad infinitas.

Por su parte, un fenómeno relacionado con la atención es la concentración, el cual se considera un proceso psíquico apoyado por el razonamiento que usa la atención y la enfoca en algo determinado. Ambas son fundamentales para aprender y ambas son requeridas de forma insistente en los centros educativos, sin que la escuela tenga, por lo general, una visión concreta de las mismas, ni mucho menos un plan concreto para su desarrollo. Desde el origen diverso con el que la atención y concentración llegan a la escuela, esta última por lo general no promueve el desarrollo de las mismas de forma directa y concienzuda (BautistaVallejo y González Guillén, 2018). De esta forma, ambas se van desarrollando casi a su suerte en un ambiente que, en ocasiones, no es el más propicio, ni en relación a los contenidos que la escuela ofrece ni mucho menos desde el punto de vista de la metodología de enseñanza para las nuevas generaciones.

En este marco, la metaatención se torna un proceso muy importante. Se trata del conocimiento que nos permite darnos cuenta de las distracciones y poner los remedios (autorregular o controlar) para controlarlos tomando medidas correctoras.

Es, así, una de las variables más importantes en el aprendizaje, tanto en el aula como fuera de ella. Supone saber discriminar de entre todos los estímulos y la información que nos llega cuál es la verdaderamente importante, de manera que se pueda obviar lo accesorio y centrar nuestra atención y concentración en lo significativo. Por tanto, si conocemos bien nuestros mecanismos mentales, sabiendo qué nos distrae y adquirimos la capacidad de centrar nuestra atención en un objetivo concreto, estaremos promoviendo el logro de un aprendizaje efectivo (Briñas, 2017). 


\subsection{Metalectura}

La lectura es una actividad de procesamiento y feedback de la información sobre el lenguaje y el texto. Por su parte, la metalectura va más allá del simple hecho de descifrar y decodificar letras y palabras. Supone tener presente el conjunto de conocimientos que tenemos sobre la lectura y los procesos psicológicos y mentales que debemos llevar a cabo para leer.

Se trata, en este sentido, del conocimiento que se tiene sobre la lectura y las operaciones mentales implicadas en la misma: para qué se lee, qué hay que hacer para leer, qué impide leer bien, qué diferencia hay entre unos textos y otros, etc. Desde un punto de vista evolutivo, el cerebro ha adaptado su capacidad para el reconocimiento del lenguaje (Galván Celis et al., 2014).

El conocimiento de la finalidad determina cómo se regula la acción de leer. Ese conocimiento y la autorregulación son dos aspectos fundamentales de la metalectura, íntimamente relacionados. Por ejemplo, cuando se advierte (conocimiento) que un párrafo es difícil, se lee más despacio (autorregulación); si se prepara un examen, se lee con mayor atención; si la letra es muy pequeña y borrosa, se acerca más el libro.

En este sentido, una de las líneas que más controversia están creando, por cuanto que los estudios se polarizan entre aquellos que dejan pasar la influencia de las tecnologías en nuestro cerebro como algo más y aquellos que entienden que está apareciendo; por ejemplo, en este caso, un nuevo "cerebro lector", como afirma Xilin (2016).

El cerebro lector se refiere "al cerebro que lee". Esto es más que patente en los cambios que aparecen en el comportamiento y el hábito de la lectura durante la lectura digital en nuevos escenarios en donde los dispositivos electrónicos parecen hegemónicos en el acceso a la lectura, como entienden Wang (2015) y Fesel et al. (2018). Aquí, la estructura neuronal del cerebro también se ajustará y remodelará, generando nuevas conexiones neuronales, formando nuevos circuitos neuronales para adaptarse al cambio de comportamiento de lectura digital en red.

Nuestros hábitos de lectura en la pantalla y el comportamiento de navegación rápida en el proceso de lectura digital pueden causar algunos cambios en el modo de procesamiento de la lectura, como el procesamiento de información de fragmentación y el pensamiento colaborativo no lineal. Así, según Xilin (2016), los grandes impactos en la cognición podrían resumirse en tres aspectos principales: 1) aumento de la carga cognitiva; 2) reducción de los costos cognitivos del lector y desarrollo de un nuevo modelo de lectura similar al modo de "memoria delegada en internet" y 3) el hecho de presentar mayores requisitos para la capacidad de metalectura en el lector.

Con la entrada de cada vez más dispositivos en donde acceder y leer la información, el "cerebro lector" también está cambiando y el rol del lector también se cambia desde un cultivador de conocimiento a un "cazador de información". Esta es la razón por la que Xilin (2016) ve la necesidad de promover, en este entorno creciente en red y lectura digital, la capacidad de la metalectura del lector, pues tiene un profundo impacto en el rendimiento de la comprensión lectora en estos nuevos entornos.

\subsection{Metaescritura}

El objetivo primordial de la escritura es comunicar, dejar constancia de algo o persuadir a las personas, por escrito, sobre algo. Por su parte, la metaescritura es el conjunto de conocimientos que poseemos sobre la escritura. Se trata, además, de cómo el individuo regula las operaciones implicadas en la comunicación escrita. 
Estos conocimientos incluyen una serie de acciones que alimentan esta posibilidad, esto es, saber cuál es la finalidad de escribir, regular la expresión de forma que logre una comunicación adecuada, evaluar cómo y hasta qué punto se consigue el objetivo, etc. (Jiménez Rodríguez et al., 2018).

La metaescritura supone que la persona sea capaz de sintetizar y expresar sus ideas por escrito de un modo eficiente, a través de, además, diferentes modelos de escritura (Alamargot y Chanquoy, 2001).

\section{NUEVO CONTEXTO EN LA ENSEÑANZA DE LAS CIENCIAS Y TECNOLOGÍAS}

Cada día son más frecuentes las investigaciones que ponen de manifiesto la relación entre las habilidades metacognitivas y el aprendizaje de las ciencias y las tecnologías, esto último en sentido amplio (Cerezo et al., 2019; Gray et al., 2018; Romero Cuervo, 2018; Tamayo-Alzate et al., 2019; Valenzuela, 2019).

De otra parte, las apreciaciones sobre el tipo de sociedad en la que estamos son constantes. Sobre si debe ser definida como la sociedad de la inteligencia, del aprendizaje, del conocimiento, de la tecnología, etc., simplemente los distintos autores no se ponen de acuerdo (Fagerberg et al., 2012). En este momento el mundo es campo abonado de profundos cambios de naturaleza social, económica, epistémica, tecnológica y cultural, de los cuales pueden extraerse, al menos, dos consideraciones generales:

Primero, la pregunta sobre el tipo de aprendizaje que se necesita para un futuro que es, ciertamente, desconocido. Por un lado, nuestros propios sistemas de conocimiento y tecnologías están produciendo un cambio reflexivo, en el cual lo producido se presenta como catalizador de futuros cambios. Es así que este elemento reflexivo está en el nexo de unión entre el conocimiento y el cambio que se produce siempre más rápido, provocando consiguientemente una situación de cambio esencialmente desconocible (Barnett, 2018b; Barnett, 2019). La pregunta es, entonces, ¿cuál es la idea que tenemos de aprendizaje?

En este contexto, Dzib Goodin (2013a) entiende que el proceso de aprendizaje no depende de los educadores, es una función otorgada a todas las especies sobre la faz de la tierra con fines de pervivencia, con el objetivo de responder lo más adaptativamente posible ante el entorno. Es decir, el aprendizaje es un proceso personal que puede ser limitado o engrandecido por el entorno. Recientemente Barnett (2018a) ha introducido esta idea en el ámbito universitario, en el marco de la llamada "universidad ecológica”, es decir, aquella que crea un entorno o ecosistema que envuelve con múltiples, diversas y combinadas actividades al alumnado "imposible de no empapar".

Segundo, que la reflexividad construida de nuestros sistemas de conocimiento tiene repercusiones a nivel individual. Los crecientes niveles de satisfacción en conceptos, ideas y, por supuesto, discursos manejados, necesitan de individuos si ellos de cualquier forma van a dar sentido al mundo que están encarando. Es por ello que la reflexividad a nivel de cada individuo, es decir, la capacidad crítica de irse interrogando sobre el universo que nos rodea, es una necesidad para a asimilar y acomodar el nuevo orden, cosa que, por otro lado, va aconteciendo como consecuencia de las influencias que recibimos y en los entornos en que nos desempeñamos.

Esta es la razón por la que el aprendizaje para un mundo desconocido llama, dicho con pocas palabras, a un cambio ontológico y, desde luego, una nueva forma de proceder en la práctica (Barnett, 2019).

En el ámbito de las ciencias, en un momento en que se perciben cambios en la estructura de los saberes, en las formas tradicionales de organización, división y especialización del conocimiento, transformación de 
la circulación y apropiación del conocimiento y cambios en el papel social de las profesiones, es necesario abordar estas situaciones con un nuevo marco de trabajo en donde estas peculiaridades sean tenidas en cuenta (Galván-Fernández et al., 2017).

En el caso de las instituciones educativas, universitarias y no universitarias, ya se advertía que al integrarse más con los criterios cognitivos de la sociedad, estas se ven obligadas a mostrar nuevas definiciones sobre lo que se debe conocer y aprender y, en consecuencia, a tomar decisiones sobre todo ello (Barnett, 2018b; Bautista-Vallejo y González Guillén, 2018; Gray et al., 2018).

\section{EL CAMBIO CURRICULAR EN LAS INSTITUCIONES EDUCATIVAS}

Hace ya más de 25 años se empezaron a tomar las primeras decisiones en relación a la inclusión de las TIC. En el caso de España, el periodo comprendido entre los años 1985 y 1995 se puede considerar como la etapa inicial en la incorporación de las TIC.

Sin embargo, el modelo, pese a ser un movimiento original de transformación de la educación gracias a la integración de ciertas tecnologías electrónicas, representó no tanto la transformación que algunos demandaban sino, más bien, la primera puerta abierta en este recorrido. Vídeos, cámaras, TV o retroproyectores suponían el primer paso, pero su impacto iba a ser muy desigual. El libro era aún hegemónico, como hoy. Pese a todo, el uso de celulares de manera masiva y las posibilidades de la electrónica, la robótica, la informática desde la ingeniería web y las tecnologías digitales emergentes (Cabero Almenara y Fernández Robles, 2018) iban terminar de abrir las puertas para una transformación que ahora ya se anuncia y parece definitiva.

En una constante de la educación, que hoy parece haberse convertido en una necesidad, cada breve plazo de tiempo se expresa la necesidad de algo nuevo que inspire nuevos cambios en la misma. Primero fue Internet, luego vino Internet de las cosas, ahora se habla de inteligencia artificial y ciencias de la computación. Se trata de un camino que se empieza a recorrer con unos resultados imprevisibles aún, en un nuevo horizonte apetecido, pero desconocido e incierto. Para algunos autores, este es precisamente el nuevo horizonte en el que tenemos que educar (Gray et al., 2018; Zúñiga et al., 2018).

Desde el punto de vista del impacto como actividad en esta nueva mirada a la educación, aquella que parece inaugurar un tiempo nuevo, aparece la programación informática como algo relevante. Más allá de algunas experiencias aisladas, es en 2015 cuando la llamada programación informática se presenta como novedad, al menos en España. Esta actividad comenzaba en la Comunidad de Madrid en una serie de centros de Educación Secundaria con proyectos piloto. Pero, contemplada esta cuestión como marco de las distintas decisiones que se han ido tomando, pese a que la enseñanza de conceptos de ciencias de la computación en la escuela ya es reconocida como prioritaria y ha logrado altos niveles de consenso y, aunque hoy está ya extendida por todo el territorio nacional e internacional, se observa que el seguimiento y la calidad de las experiencias es, ciertamente, muy discutible. Por ejemplo, Garis et al. (2018) y Sommer et al. (2018) se refieren de forma crítica al caso argentino y Rodríguez y González (2018) al caso español.

Con relación al caso concreto de la programación informática en las escuelas es en los años 80 cuando se lanzó el lenguaje de programación Logo en miles de estas, principalmente en Estados Unidos. Estas escuelas comenzaron a introducir la programación en sus currículos. Al tiempo que las escuelas extendían el uso de esta nueva actividad, la investigación les acompañaba con el interés puesto en conocer cuál era el impacto de la programación en el alumnado (Clements, 1986; Grover, 2019). 
Así, en el año 1986, el artículo de D. H. Clements "Effects of Logo and CAI environments on cognition and creativity" explicaba en sus conclusiones que los niños y niñas que usaron Logo en Educación Infantil demostraron mayor capacidad de atención, más autonomía y mostraban un mayor placer por el descubrimiento de nuevos conceptos.

Desde entonces los estudios continuaron y siguieron las demostraciones, esta vez de la mano de programas como Scratch, probablemente el software para programar en edades de 5 a 7 años más usado a nivel internacional (Oda y Horita, 2019; Tsai et al., 2019). Estas investigaciones llegan a las siguientes conclusiones: el alumnado que participa en estas actividades demostró obtener mejores resultados en pruebas de matemáticas, razonamiento y resolución de problemas, todas ellas llamadas habilidades de pensamiento (thinking skills) (Bers, 2018). Es de esta forma, con el desarrollo del llamado pensamiento computacional (Grover, 2019), como la programación se convierte en una nueva forma de alfabetización, tal vez la más importante para el futuro, en donde, según Bers (2018), las TIC se convierten en "patio de recreo" en donde se anima a niños y niñas a una exploración con mente abierta de la realidad, a la creatividad, a la imaginación y a las interacciones sociales, así como a adquirir habilidades, maestría y capacidad de resolución de problemas.

En definitiva, lo que los estudios más recientes demuestran, por ejemplo García-Peñalvo y Mendes (2018) o Grover, Domínguez et al. (2019), es la importancia de aprovechar el acceso a las ciencias de la computación, es decir, introducir pronto al alumnado en todo aquello que tiene que ver con la informática, la generación de código y, también, la robótica, en aulas de infantil, primaria y secundaria, fundamentalmente en etapas tempranas, para promover el pensamiento computacional de los jóvenes estudiantes, la motivación en temas de ciencia y tecnología (Solé-Llussà et al., 2019) y el aprendizaje de habilidades básicas como la colaboración, la persistencia, la abstracción y la creatividad para tener éxito en el mundo digital de hoy.

Esto está llevando, y llevará de forma creciente, a la toma de decisiones en el ámbito curricular. En el contexto internacional, que cada vez más sirve de referencia, el Informe Horizon (Adams et al., 2017) presenta las tendencias clave en la adopción de tecnologías en educación que se consideran con un elevado potencial, a saber:

- A corto plazo: la alfabetización en programación y el aumento del aprendizaje STEAM (a diferencia de STEM es aquella que incluye, también, un enfoque basado en las artes).

- A medio plazo: el interés creciente en la medición del aprendizaje y el rediseño de los espacios de aprendizaje (los que han sido denominados por Barnett espacios de instituciones ecológicas).

- A largo plazo: avances en la cultura de la innovación y los enfoques de aprendizaje profundo.

\section{CONCLUSIONES}

A lo largo de estas líneas hemos realizado un recorrido por los distintos elementos que forman parte del proceso de metacognición. Hasta ahora, la preponderancia de una concepción de la inteligencia basada en el cociente intelectual definido por Binet y Simon, condicionó durante décadas la percepción que el docente tenía de su alumnado sesgando, en muchos de modo negativo, las expectativas que tenía sobre su proceso de aprendizaje e influyendo, también, en su auto-concepto y autoestima académica.

A partir del análisis realizado, es posible reconocer que la relación entre las actividades y entornos generados desde un modelo STEM se encuentran íntimamente relacionadas con el proceso metacognitivo, 
evidencias que se muestran tanto en el plano cognitivo como neurológico (Perry et al., 2018; Snow y Kaplan, 2018; Tamayo-Alzate et al., 2019; Tanner, 2012).

Gracias a la evidencia neuro-cognitiva y porque el conocimiento metacognitivo desempeña un papel fundamental en la selección y regulación inteligente de estrategias y técnicas de aprendizaje, algunos investigadores han analizado estrategias relacionadas con el desarrollo de habilidades metacognitivas a partir de las habilidades adquiridas en un contexto de aprendizaje integrado STEM, ejemplo de ello es el trabajo realizado por Briñas (2017) y Romero Cuervo (2018).

Sin embargo, no debe perderse de vista que la cultura y el entorno cultural juegan un papel relevante incluso en la conformación de las estructuras cerebrales debido a la plasticidad cerebral, como confirma Dzib Goodin (2013b). Esto implica que la aplicación de este tipo de estrategias no puede ser discriminada y que el fracaso de las mismas no puede ser razonado como un fallo por parte de los participantes, sino algo que puede deberse a la elección de los estímulos.

Una vez definidos y analizados estos componentes del proceso metacognitivo, es primordial que el ambiente diseñado permita al alumnado ser consciente de cuáles son las estrategias que mejor les ayudan a aprender, de manera que sean capaces de poner en práctica la metacognición, o, dicho de otro modo, que sean capaces de conocer su propia memoria, de qué manera aprenden con más eficacia, de qué forma comprenden mejor. Que aprendan a aprender por sí mismos, según sus propios esquemas conceptuales, partiendo de sus conocimientos previos, lo cual les va a permitir dotar de significatividad y profundidad sus aprendizajes. La planificación didáctica realizada por los docentes debe tener presente todos estos aspectos metacognitivos de manera que puedan personalizarse, igualmente, los procesos de enseñanza-aprendizaje.

Se reconoce, finalmente, que la aplicación de un modelo STEM en las aulas no puede verse como algo restringido ya que esto ayuda al desarrollo de habilidades asociadas, como el lenguaje, la activación motora, los procesos metacognitivos y de memoria, los cuales son parte de los entornos culturales de niños y niñas y con ellos desarrollan sus propias capacidades para poder responder con ellas en el medio ambiente en que se desarrollan.

\section{REFERENCIAS}

Adams, S., Cummins, M., Davis, A., Hall, C. y Freeman, A. (2017). The NMC/CoSN Horizon Report: 2017 K - 12 Edition. The New Media Consortium.

Agra, G., Formiga, N.S., Oliveira, P.S.D., Costa, M.M.L., Fernandes, M.D.G.M. y Nóbrega, M.M.L.D. (2019). Analysis of the concept of Meaningful Learning in light of the Ausubel's Theory. Revista brasileira de enfermagem, 72(1), 248-255.

Aguilera Morales, D., Martín-Páez, T., Valdivia-Rodríguez, V., RuizDelgado, Á., Williams-Pinto, L., Vílchez-González, J.M. y Perales-Palacios, F.J. (2018). La enseñanza de las ciencias basada en indagación. Una revisión sistemática de la producción española Inquiry-based Science Education. A systematic review of Spanish production. Revista de Educación, 381, 259-284.
Alamargot, D. y Chanquoy, L. (2001). Through the Models of Writing. Kluwer Academic Press.

Allman, M.J. y Mareschal, D. (2016). Possible evolutionary and developmental mechanisms of mental time travel (and implications for autism). Current Opinion in Behavioral Sciences, $8,220-225$.

Arabit García, J. y Prendes Espinosa, M.P. (2020). Metodologías y Tecnologías para enseñar STEM en Educación Primaria: análisis de necesidades. Píxel-Bit. Revista de Medios y Educación, 57, 107-128.

Barnett, R. (2018a). The Ecological University: a university whose time has come. In: Ronald Barnett y Michael Peters (eds). 
The Idea of the University (pp. 141-151). Vol. 2, Contemporary Perspectives. Peter Lang.

Barnett, R. (2018b). The University after Postmodernism: An Ecological Approach. Educational Philosophy and Theory, 50(14) 1537-1538.

Barnett, R. (2019). The Thoughtful University: A Feasible Utopia. Beijing International Review of Education, 1(1) 55-72. https:// doi.org/10.1163/25902547-00101007

Bautista-Vallejo, J.M. y González Guillén, F. (2018). Los secretos del fracaso escolar. Amazon.

Bers, M.U. (2018). Coding as a playground. Programming and Computational Thinking in the Early Childhood Classroom. Routledge.

Briñas, L.T. (2017). Priorizar la competencia aprender a aprender en las programaciones y unidades didácticas. International Journal of Developmental and Educational Psychology. Revista INFAD de Psicología, 7(1), 131-140.

Cabero Almenara, J. y Fernández Robles, B. (2018). Las tecnologías digitales emergentes entran en la Universidad: RA y RV. RIED. Revista Iberoamericana de Educación a Distancia, 21(2), 119-138.

Cerezo, R., Fernández, E., Amieiro, N., Valle, A., Rosário, P. y Núñez, J.C. (2019). El papel mediador de la autoeficacia y la utilidad entre el conocimiento y el uso de estrategias de autorregulación del aprendizaje. Revista de Psicodidáctica, 24(1), 1-8.

Clements, D. (1986). Effects of Logo and CAl environments on cognition and creativity. Journal of Educational Psychology, 78(4), 309-318.

De Bruin, A.B.H., Thiede, K.W., Camp, G. y Redford, J. (2011). Generating keywords improves metacomprehension and selfregulation in elementary and middle school children. Journal of Experimental Child Psychology, 109(3) 294-310. https://doi. $\operatorname{org} / 10.1016 /$ j.jecp.2011.02.005

Dzib Goodin, A. (2013a). La evolución del aprendizaje más allá de las redes neuronales. Revista Chilena de Neuropsicología, $8(1), 20-25$

Dzib Goodin, A. (2013b). La arquitectura cerebral como responsable del desarrollo del proceso de aprendizaje. Revista Mexicana de Neurociencia, 14(2), 81-85.
Dzib-Goodin, A., Sanders, L. y Yelizarov, D. (2017). Sistemas Neuro-Moleculares necesarios para el proceso de memoria. Cuadernos de Neuropsicología/Panamerican Journal of Neuropsychology, 11(1), 82-102.

Eysenck, H. (2018). Intelligence: A new look. Routledge.

Fagerberg, J., Landström, H. y Martin, B.R. (2012). Exploring the emerging knowledge base of 'the knowledge society'. Research Policy, 41, 1121-1131. https://doi.org/10.1016/j.respol.2012.03.007

Fesel, S.S., Segers, E. y Verhoeven, L. (2018). Individual variation in children's reading comprehension across digital text types. Journal of Research in Reading, 41(1), 106-121.

Flavell, J. (1976). Metacognitive aspects of problem solving. En L. Resnick (Ed.), The nature of intelligence (pp. 231-236). Lawrence Erlbaum.

Flavell, J. (1979). Metacognition and cognitive monitoring: a new area of cognitive-developmental inquiry. American Psychologist, 34(10), 906-911.

Flavell, J. (1992). Desarrollo cognitivo: pasado, presente y futuro. Developmental Psychology, 28(6), 998-1005.

Galván Celis, V., Mikhailova Pechonkina, I. y Dzib Goodin, A. (2014). La relación entre los procesos de lecto-escritura y la música desde la perspectiva neurocognitiva. Revista Chilena de Neuropsicología, 9(1-2), 21-24.

Galván-Fernández, C., Rubio-Hurtado, M., Martínez-Olmo, F. y Rodríguez-Illera, J. (2017). Can the integration of a PLE in an e-portfolio platform improve generic competences? Journal of New Approaches in Educational Research (NAER Journal), $6(2), 112-118$.

García-Peñalvo, F.J. y Mendes, A.J. (2018). Exploring the computational thinking effects in pre-university education. Computers in Human Behavior, 80, March, 407-411.

Gargallo López, B., Pérez-Pérez, C., García-García, F.J., Giménez Beut, J.A. y Portillo Poblador, N. (2020). La competencia aprender a aprender en la universidad: propuesta de modelo teórico. Educación XX1, 23(1), 19-44. https://doi.org/10.5944/ educXX1.23367

Garis, A.G., Albornoz, M.C. y Silvestri, M.A. (2018). Analizando el impacto de talleres de programación en escuelas con respec- 
to al ingreso de alumnos en carreras de informática. En $X X$ Workshop de Investigadores en Ciencias de la Computación (WICC 2018, Universidad Nacional del Nordeste).

Godhe, A.L., Lilja, P. y Selwyn, N. (2019). Making sense of making: critical issues in the integration of maker education into schools. Technology, Pedagogy and Education, 1-12. https:// doi.org/10.1080/1475939X.2019.1610040

Gray, S.L., Scott, D. y Mehisto, P. (2018). Belonging Together: A Model for Education in a New European Age (pp. 139159). En P. Mehisto, S.L. Gray y D. Scott: Curriculum Reform in the European Schools. Palgrave Macmillan. https://doi. org/10.1007/978-3-319-71464-6_7

Grover, S. (2019, February). Thinking about Computational Thinking: Lessons from Education Research (pp. 1283-1283). En Proceedings of the 50th ACM Technical Symposium on Computer Science Education. ACM.

Grover, S., Dominguez, X., Kamdar, D., Vahey, P., Moorthy, S., Rafanan, K. y Gracely, S. (2019, February). Integrating Computational Thinking in Informal and Formal Science and Math Activities for Preschool Learners (pp. 1257-1258). En Proceedings of the 50th ACM Technical Symposium on Computer Science Education. ACM.

Jiménez Rodríguez, V., Alvarado Izquierdo, J.M. y Calaforra Faubel, P.J. (2018). Metacognitive Strategies Applied to Writing as Predictors of Spontaneous Writing Quality. Electronic Journal of Research in Educational Psychology, 16(45), 301-322.

Llewelyn, S. y Hobson, J.A. (2015). Not only... but also: REM sleep creates and NREM stage 2 instantiates landmark junctions in cortical memory networks. Neurobiology of learning and memory, 122, 69-87.

López Mejías, M.; Cuenca Díaz, M. y Cabrera Hernández, Y. (2017). La metamemoria: un recurso de aprendizaje básico en el ámbito escolar. Transformación, 13(1), 43-55.

Metcalfe, J. y Dunlosky, J. (2008). Metamemory. En H. Roediger (Ed.). Cognitive psychology of memory (pp. 349-362). Elsevier. Murcia, K. y Pepper, C. (2018). Evaluating the social impact of a science centre's STEM professional learning strategies for teachers. Issues in Educational Research, 28(2), 438-452.

Oda, M. y Horita, T. (2019, March). Characteristics and Challenges of Japanese Computer Science Education in Elementary Le- vel (pp. 62-69). En Society for Information Technology \& Teacher Education International Conference. Association for the Advancement of Computing in Education (AACE).

Ott, D.L. y Michailova, S. (2018). Cultural intelligence: A review and new research avenues. International Journal of Management Reviews, 20(1), 99-119.

Perry, J., Lundie, D. y Golder, G. (2018). Metacognition in schools: what does the literature suggest about the effectiveness of teaching metacognition in schools? Educational Review, 1-18. https://doi.org/10.1080/00131911.2018.1441127

Riley, M.R. y Constantinidis, C. (2016). Role of prefrontal persistent activity in working memory. Frontiers in Systems Neuroscience, 9. https://doi.org/10.3389/fnsys.2015.00181.

Rodríguez Palmero, M.L. (2004) Teoría del Aprendizaje Significativo (pp. 535-544). En Concept Maps: Theory, Methodology, Technology. Proc. of the First Int. Conference on Concept Mapping. Universidad Pública de Navarra, Pamplona, Spain.

Rodríguez, S.D. y González, M.F. (2018). Análisis de la implantación de las TIC en la Educación Secundaria. Tendencias tecnológicas actuales. Revista de Estilos de Aprendizaje, 11(22), 109-135. http://revistaestilosdeaprendizaje.com/article/ view/1082

Romero Cuervo, E.M. (2018). Ambiente multimodal de aprendizaje para el fomento de la compresión lectora de textos explicativos en ciencias naturales (Master's thesis, Universidad de La Sabana, Colombia)

Sivaraj, R., Ellis, J. y Roehrig, G. (2019, March). Conceptualizing the T in STEM: A Systematic Review (pp. 991-1000). En Society for Information Technology \& Teacher Education International Conference. Association for the Advancement of Computing in Education (AACE).

Snow, E. y Kaplan, M. (2018). The New Five-Year Federal Strategic Plan in STEM Education: What's in it for Science? En AGU Fall Meeting Abstracts, December.

Solé-Llussà, A., Aguilar Camaño, D. y Ibáñez Plana, M. (2019). Las ayudas en indagaciones científicas escolares mediadas por herramientas tecnológicas. Investigaciones de la última década. Digital Education Review, 36, 223-242.

Sommer, S., Cornejo, M.E., Cortez, M. y Rodríguez, J. (2018). El lugar de las Ciencias de la Computación en el currículum de la 
escuela secundaria argentina. En XX Workshop de Investigadores en Ciencias de la Computación (WICC 2018, Universidad Nacional del Nordeste).

Soodla, P.; Jõgi, A.L. y Kikas, E. (2017). Relationships between teachers' metacognitive knowledge and students' metacognitive knowledge and reading achievement. European Journal of Psychology of Education, 32(2), 201-218.

Spruce, R.; Bol, L. (2015). Teacher beliefs, knowledge, and practice of self-regulated learning. Metacognition and Learning, 10, 245-277.

Tamayo-Alzate, Ó.E., Cadavid-Alzate, V. y Montoya-Londoño, D.M. (2019). Análisis metacognitivo en estudiantes de básica, durante la resolución de dos situaciones experimentales en la clase de Ciencias Naturales. Revista Colombiana de Educa ción, 76, 117-141.

Tanner, K. (2012). Promoting student metacognition. CBE Life Sciences Education, Bethesda, 11(2), 113-120.

Tsai, M.-J., Wang, C.-Y. y Hsu, P.-F. (2019). Developing the Computer Programming Self-Efficacy Scale for Computer Literacy Education. Journal of Educational Computing Research, 56(8), 1345-1360. https://doi.org/10.1177/0735633117746747

Tulving, E. y Madigan, S. (1970). Memory and verbal learning. Annual Review of Psychology, 21(1), 437-484.

Valenzuela, Á. (2019). ¿Qué hay de nuevo en la metacognición? Revisión del concepto, sus componentes y términos afines. Educação e Pesquisa, 45, e187571-e187571. DOI: https://doi. org/10.1590/s1678-4634201945187571

Villota Hurtado, O. (2018). Uso del portafolio digital como herramienta cognitiva. Modelo para una evidencia significativa. EDMETIC, 7(1), 321-349. https://doi.org/10.21071/edmetic. v7i1.5751

Wang, S. (2015). On Digital Reading. Library Journal, 34(4), 4-10.

Wellman, H. (1985). The origins of metacognition (1-30). En Forrest Presley, D.; Mackinton, G.; Waller, G. (Ed.). Metacognition, cognition and human perfomance. Academic Press.

Xilin, Y. (2016). Digital Reading Behavior \& Habit Can Affect "Reading Brain" and Cognitive Model of Reading. Libraly Journal, 35(4), 18-26.
Zepeda, C., Richey, J. E., Ronevich, P. y Nokes-Malach, T. (2015). Direct Instruction of metacognition benefits adolescent science learning, transfer, and motivation: an in vivo study. Journal of Educational Psychology, 107(4), 954-970.

Zúñiga, R.P., Lozano, P.M., García, M.M., Hernández, E.M. y Ibarra, J.Á.P. (2018). La sociedad del conocimiento y la sociedad de la información como la piedra angular en la innovación tecnológica educativa. RIDE Revista Iberoamericana para la Investigación y el Desarrollo Educativo, 8(16), 847-870. 